\title{
Initial results for dual constellation dual-frequency multipath models
}

\author{
Mihaela-Simona Circiu, Michael Felux, Stefano Caizzone, Christoph Enneking, Friederike Fohlmeister, Markus Rippl (German \\ Aerospace Center) \\ Ioana Gulie, David Rüegg (Airbus Defence and Space) \\ Joseph Griggs (Collins Aerospace) \\ Rémy Lazzerini, Florent Hagemann, Francois Tranchet (Airbus Operation SAS) \\ Pierre Bouniol (Thales Avionics) \\ Matteo Sgammini (Joint Research Center of the European Commision)
}

\section{BIOGRAPHY (IES)}

Mihaela-Simona Circiu received a Bachelor and Master's Degree in Computer Engineering from Technical University Gheorghe Asachi, Romania. In 2012, she obtained a 2nd level Specialized Master in Navigation and Related Application from Politecnico di Torino, Italy. She joined the German Aerospace Center in 2013 where she is working on the development of the multi-frequency multi-constellation Ground Based Augmentation System (GBAS) and multipath models.

Dr. Michael Felux is a research associate at the German Aerospace Center (DLR). He graduated in technical mathematics at Technische Universität München (TUM) in 2009 and received his Doctoral Degree in Mechanical Engineering also from the TUM in 2018. Since joining DLR in 2009 he is specializing on aeronautical navigation integrity with a special focus on GBAS for CATII/III operations. He is active in different GBAS working groups and is program manager for the research on GBAS navigation in DLR since 2015.

Dr. Stefano Caizzone received the M.Sc. in Telecommunications Engineering and the Ph.D. degree in Geoinformation from the University of Rome "Tor Vergata", Italy, in 2009 and 2015, respectively. Since 2010, he is with the Antenna group of the Institute of Communications and Navigation of the German Aerospace Center (DLR), Wessling, Germany, where he is responsible for the development of innovative miniaturized antennas. His main research interests concern small antennas for RFIDs and satellite navigation, antenna arrays and grids with enhanced sensing capabilities, controlled radiation pattern antennas for robust satellite navigation and high-performance antenna design for precise satellite navigation. He is a participant of RTCA SC-159, active in the definition of minimum operational performance specifications for avionic GNSS equipment.

Christoph Enneking received the BSc. and the MSc. degrees in electrical engineering from the Munich University of Technology (TUM), Germany, in 2012 and 2014, respectively. In September 2014, he joined the Institute of Communications and Navigation of the German Aerospace Center (DLR), Wessling-Oberpfaffenhofen. His research interests include GNSS signal design, estimation theory, and GNSS intra- and intersystem interference.

Friederike Fohlmeister received the BSc. and the MSc. degrees in electrical engineering from Technische Universität München (TUM) in 2012 and 2014, respectively. In December 2014, she joint the Institut of Communications and Navigation of the German Aerospace Center (DLR). Her research interests include GNSS signal processing under multipath, ionospheric scintillations and other disturbances.

Markus Rippl received his Diploma in Electrical Engineering and Information Technology from Technische Universität München (TUM) in 2007. Since then, he has been a research associate with the Institute of Communications and Navigation (IKN) at the German Aerospace Center (DLR) in Oberpfaffenhofen near Munich. His areas of interest include GNSS integrity using receiverside algorithms, next generation Receiver Autonomous Integrity Monitoring (RAIM) algorithms and architectures, and the development of a future integrity architecture supporting Advanced Receiver Autonomous Integrity Monitoring (ARAIM) with an Integrity Support Message (ISM).

Ioana Gulie received her degree in Electronics, Telecommunications and Information Technology from University Politehnica of Bucharest, Romania in 2007. She obtained the Master of Science (MSc) in Engineering from Aalborg University, Denmark in 2014. She is working for the department of Navigation Products at Airbus Defence and Space GmbH since 2015. She is involved in research and development activities such as software receiver development projects, with focus on tracking and multipath mitigation methods, multipath channel modelling and interference localization activities.

David Rüegg received his M.Sc. in Electrical Engineering from the Karlsruhe Institute of Technology, Germany, in 2017. In 2018 he joined Airbus Defence and Space GmbH in Munich, where he is mainly involved in the development and implementation of navigation and positioning algorithms for GNSS receivers on various levels from tracking to PVT, as well as in activities related to SBAS and integrity. 
Joseph Griggs received a BEng Degree in Computer System Engineering from Brunel University London in 2017. In 2017, he joined Collins Aerospace as a Systems Engineer, working on various GNSS projects in areas such as integrity algorithms for aviation, spoofing detection techniques and high precision inertial and GNSS targeting systems.

Rémy Lazzerini received a Master's Degree in Computer Engineering from Conservatoire National des Arts et Métiers in 2010 and a Specialized Master in Telecommunication and Navigation from ISAE (Institut Supérieur de l'Aeronautique et de l'Espace) in 2013. After an early career in Computer and Telecommunication systems, he joins Airbus Commercial Aircraft where he currently manages Radio Navigation and Data Science projects.

Florent Hagemann received a Bachelor Degree in Electrical Engineering from Montpellier University and Master's Degree in Aeronautical Engineering and Maintenance from Bordeaux University in 2009. After a career in laboratory team for Surveillance systems, he joins the Radio Navigation and Surveillance Design Office.

Francois Tranchet received a MSc. degree in Navivation, radio and communication sytems from ISAE - ENSICA in 2008. He joined Airbus in 2012 and he is working now as a GNSS expert. He is chairing the Multi-constellations and Multi-frequencies adhoc group of the EUROCAE WG-62 Galileo standardization group.

Pierre Bouniol joined Thales in 2006. After managing the Navigation System Engineering team and the GNSS Products development team in Valence-France, Pierre is now in charge of the GNSS receivers product strategy. The GNSS product line is composed of four product families: GNSS receivers for military applications, civil certified GNSS receivers, anti-jamming equipment, and ground station reference/monitoring receivers. He is also the chairman of the EUROCAE WG62 "Galileo" since 2010, in charge of standardizing the use of Galileo in civil certified receivers. Pierre is also the president of the French institute of navigation, the IFN.

Matteo Sgammini is a technical officer at the Joint Research Centre (JRC) of the European Commission. He was a system and software engineer at MTU Aero Engines from 2006 to 2008. From September 2008 to April 2017 he was a research associate in the Navigation group of the Institute of Communication and Navigation at the German Aerospace Center (DLR), Germany. His research includes signal processing and estimation theory for GNSS. Currently he focuses on GNSS integrity and Galileo system performance verification

\section{ABSTRACT}

This paper presents an update of the ongoing work to develop dual frequency dual constellation airborne multipath models for Galileo E1, E5a and GPS L1 and GPS L5 in the frame of the project DUFMAN (Dual Frequency Multipath Models for Aviation) funded by the European Commission. The goal of this activity is to support the development and implementation of airborne GNSS-based navigation solutions, such as Advanced Receiver Autonomous Integrity Monitoring (ARAIM), dual-frequency multiconstellation Satellite Based Augmentation System (SBAS) and dual-frequency multi-constellation Ground based Augmentation System (GBAS).

Previous work described the methodology proposed to derive the airborne multipath models and presented preliminary multipath models obtained from an experimental installation.

In this paper we present the initial results obtained from flight campaigns conducted within DUFMAN on Airbus commercial aircraft. The measurements are collected from prototypes of dual-frequency multi-constellation avionics receiver and the antenna installed on the aircraft has been selected to meet at best the current dual-frequency dual-constellation antenna requirements.

In addition to the initial results obtained from avionics hardware, the impact of the different receiver correlator spacing and bandwidth is investigated and discussed.

\section{INTRODUCTION}

Aeronautical navigation is increasingly based on the use of Global Navigation Satellite Systems (GNSS). It is a cornerstone of performance based navigation and enables airspace users to ensure that their navigation capabilities meet the required level of performance. For different phases of flight, different levels of integrity, accuracy and availability are required. The requirements for position errors reach from rather generous error budgets on the order of nautical miles horizontally with a $95 \%$ probability for the en-route phase down to 10 meters vertical with an integrity risk of just $10^{-7}$ for precision approaches. Different techniques and augmentation systems, such as (advanced) receiver autonomous integrity monitoring (A)RAIM and space and ground based augmentation systems (SBAS, GBAS) continue to be developed in order to be able to satisfy all performance requirements for current and future airspace usage. 
On the side of the GNSS space segments, the European Galileo constellation continues to grow and has now 22 operational satellites available. All the Galileo satellites offer signals on two frequencies (E1 and E5a) usable for aeronautical navigation. The US GPS constellation offers the L1/L5 dual-frequency capability on all satellites of the latest generation (Block IIF), of which 12 are in orbit and available for navigation. All new satellites from the Block IIIA generation (2 satellites already in orbit, the rest due for launch in the coming years) will also include the L5 signals.

The ARAIM concept exploits the use of dual frequency measurements to remove the ionospheric delay, the European SBAS EGNOS will provide augmentation for the Galileo and GPS constellations and for two frequencies in its V3 phase and on the GBAS side the development of dual frequency and multi-constellation techniques to cope with challenges posed by the active ionosphere in equatorial regions is ongoing as well. All GNSS-based navigation methods need to bound any potential residual position errors. One important contributor to the positioning error is the airborne multipath, i.e. signal reflections from the airframe. The impact of multipath on the GPS L1 signal was characterized long time ago and appropriate error models are used for integrity purposes ([1],[2]). However, no such models exist for GPS L5 and all Galileo signals. The modulation of the GPS L5 signal, as well as the Galileo E5a signal has a 10 times higher chipping rate than the GPS L1 signal and should therefore be less susceptible to multipath. The Galileo E1 signal uses a different modulation than the GPS L1 signal and may therefore also show different multipath characteristics. When combining the measurements on both frequencies to eliminate the first-order ionospheric delay, also the multipath contained in both measurements is combined and becomes the dominant error source and thus needs to be bounded appropriately.

In order to close this gap in the error models, multipath error models for GPS L5 and both Galileo signals are currently being developed within the DUFMAN (Dual Frequency Multipath Models for Aviation) project based on a combination of GNSS data collected in flight tests from different aircraft, different antennas and receiver configuration as well as electromagnetic and receiver simulations. The project is funded by the European Commission and is executed by a consortium which includes the German Aerospace Center (DLR), Airbus Civil Aircraft, Airbus Defense and Space, Collins Aerospace and Thales Avionics. The paper presents the ongoing work and the results of the studies performed to date within the project shows preliminary versions of the dual-frequency dual constellation multipath model for aviation. The studies include the investigation of the impact of different airframes and different receiver configurations.

In addition to the measurement-based models described in this paper, electromagnetic simulations are performed to predict the multipath model by simulation. The methodology to derive these simulation-based models is presented in a concurrent paper at the conference. In that paper a comparison between the measurement-based and simulation-based models will be included.

\section{PROJECT OVERVIEW}

The scope of the project is to develop airborne multipath models for GPS L5 and Galileo E1 and E5a based on one side on GNSS airborne measurements and on the other side, to perform a detailed analysis of the contributors to the multipath error, such as airframe, antenna and receiver parameters.

In an initial phase towards developing the airborne multipath models around 200 hours of experimental flight data collected with an experimental hardware installed on DLR's A320 research aircraft was analyzed. The methodology was presented in detail in [3] and preliminary results collected with this experimental installation were shown in [4]. The previously derived versions of the models were more conservative and exceeded the existing $\sigma_{\text {air }}$ models for GPS L1. However, the values were not to be considered final values as they were derived based on the experimental installation on a single aircraft and they were used to establish and validate the methodology for deriving multipath models. The equipment used in the experimental data collection was not compliant with the dual-frequency MOPS which led to worse results than what is expected from avionics compliant hardware. The antenna used in the experimental data collection was non-compliant with dual-frequency antenna MOPS [5] in terms of antenna group delay variations which led to worse results than what is expected from a MOPS compliant antenna. In addition the antenna group delay variations, the antenna multipath rejection capability plays an important role in how much multipath is received. As the used antenna has a high axial ratio, this effect is reflected in the measurements and the obtained results. Furthermore, the antenna was not installed in its primary location, but in an experimental location further to the back of the aircraft. In this location, the antenna was closer to other reflectors and thus the amount of multipath that is received is expected to be higher.

In order to extend the initial studies, a flight test campaign for collection of data from a variety of Airbus passenger aircraft is performed within the project. The Airbus flight test campaign uses commercially available multiband antennas in the original 
antenna locations on the airframe. Before the installation on the aircraft each antenna is measured and characterized in an anechoic chamber in order to ensure its compliance with current antenna MOPS in terms of group delay variations and axial ratio.

This paper presents the initial results from the first measurement campaign conducted on A321. During this campaign, base band samples were recorded with a bitgrabber. The samples were replayed in the lab to the Collins Aerospace GLU-2100dual-frequency multi-constellation (DFMC) prototype through an Averna replay device. The prototype receiver is capable of tracking GPS and Galileo signals on L1/E1 and L5/E5a and is a representative airborne avionics GNSS receiver. In addition, the RF-data was replayed to the Javad receiver used in the DLR's experimental installation, allowing a comparison of the results. Furthermore, studies of variations of receiver parameters, such as correlator spacing and receiver bandwidth were carried out and the obtained results are discussed in this paper.

During the upcoming months, further measurements data will be gathered in test flights of the A320, A330 and A350 aircraft.

\section{DATA COLLECTION}

On $14^{\text {th }}$ and $15^{\text {th }}$ February 2019 , the first flight campaign that provided data to the DUFMAN project took place using an Airbus A321. In the configuration of this aircraft, a multiband antenna was installed in one of the primary GNSS antenna locations (GPS2 position) as shown in Figure 1.

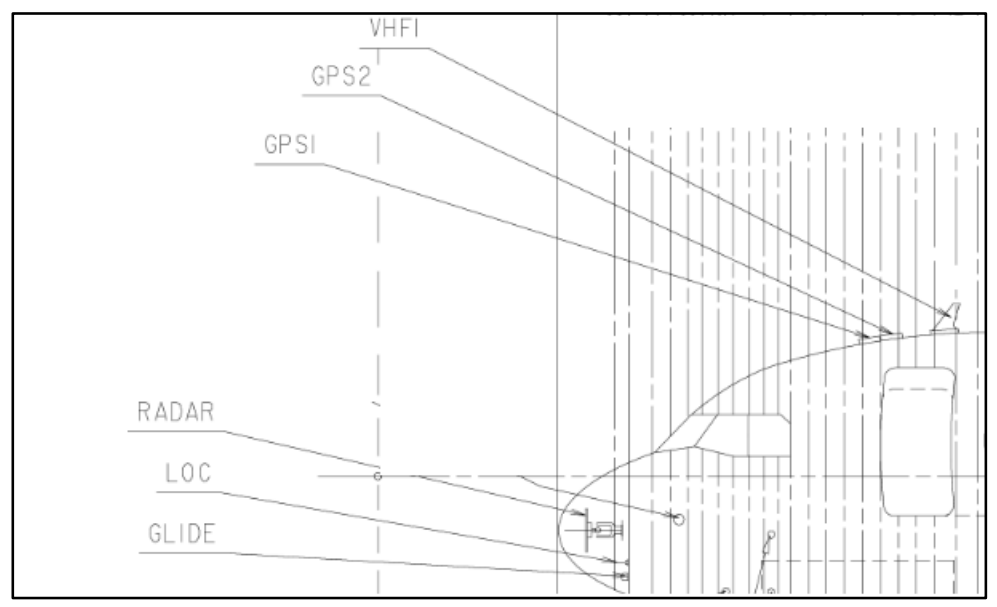

Figure 1 - Antenna location on A321

Dual-frequency GPS and Galileo base band samples were collected by the onboard Syntony ECHO-R recording device. A total of approximately 5 hours of RF samples were recorded during two flights around Toulouse Blagnac airport. Figure 2 shows the ground track of the two flights. Flight F0324 was conducted mostly south of Toulouse in a general East-West direction with some steep manoeuvring and one take-off and landing. The flight was conducted mainly between flight levels 100 and 150 and speeds between about 130 knots and 220 knots ground speed. During the second flight (F0325), 5 patterns around Toulouse Airport were flown. Each of the approaches was completed by a landing, followed by a period of taxiing back to the beginning of the runway, some stationary time at the hold position and a subsequent standard take-off, or taxiing to the parking position at the end of the flight. 


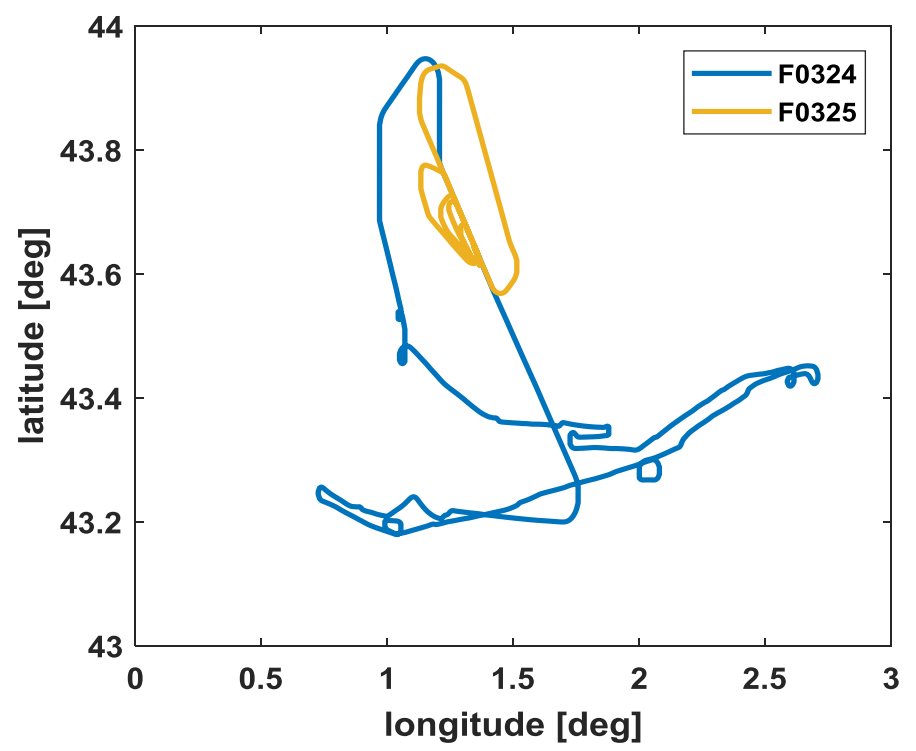

Figure 2 - Ground tracks of the flights conducted on A321

in February 2019

\section{HARDWARE USED IN THE FLIGHT CAMPAIGN}

\section{Antenna installed on the Airbus aircraft}

The antenna installed on the Airbus aircraft was measured for characterization in the spherical near-field measurement chamber available at DLR. For the measurements, the antenna was mounted on a circular rolled edges ground plane to better approximate the conditions of the antenna installed on the aircraft ([6]). The obtained pattern of the antenna was exported as a function of frequency, elevation and azimuth angles. These measurements are used on one hand to calculate the code errors introduced by the antenna itself and on the other hand as input in the electromagnetic simulations. Figure 3 shows the code errors introduced by the antenna group delay on GPS L1(top left plot), Galileo E1 (top right plot) and GPS L5/Galileo E5a (bottom graph) signals. The code errors are calculated using the antenna transfer function and an ideal receiver as described in detail in [6] and [7]. Note that the values refer to the absolute group delay (not normalized) and thus the error for each frequency and modulation has a different initial value. The total variation is kept to $1 \mathrm{~m}$ for all three graphs. It can be observed that the code errors on L1/E1 show less variations compared to L5/E5a, meaning that this antenna is optimized for the L1 band. More details on the results and the performance of the antenna are presented [8] (where this antenna is referred to as Antenna \#2). 

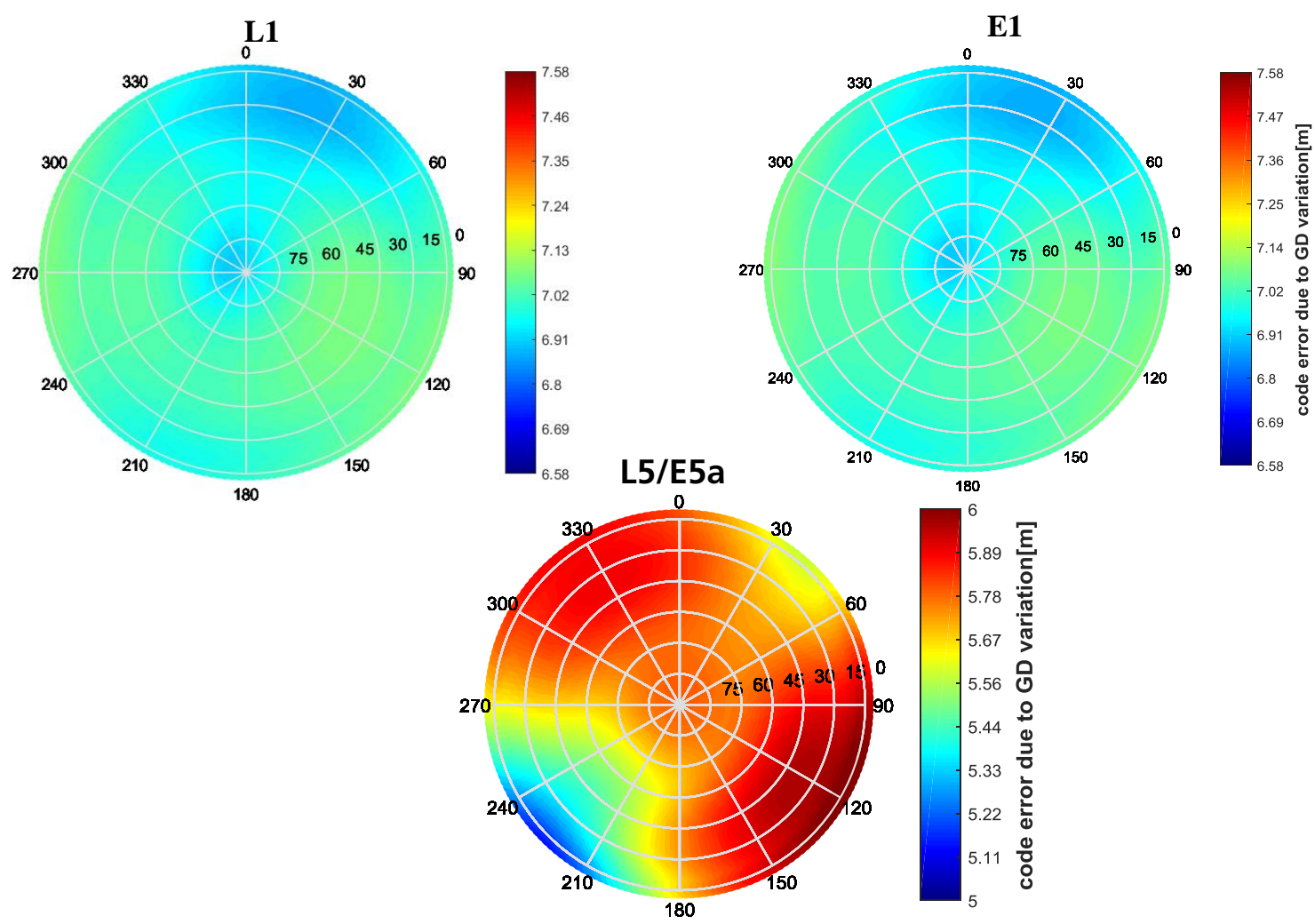

Figure 3 - Code tracking error for the antenna installed on Airbus aircraft for GPS L1 BPSK(1) signal (top left plot), Galileo E1 BOC(1,1) signal (top right plot) and GPS/Galileo L5/E5a BPSK(10) signal (bottom plot)

\section{Receivers}

The RF-samples were replayed through the Collins GLU 2100 DFMC prototype which is based on the existing production GLU2100 Multi-Mode Receiver. The prototype software allows the receiver to track GPS L5, Galileo E1 B (BOC $(1,1)$ ) and E5a signals in addition to the legacy GPS L1. The measurements are output with a $5 \mathrm{~Hz}$ sampling rate. The receiver uses the early, prompt and late correlators which were setup for these initial tests to 0.1 chips for L1/E1 signals and 1 chip for L5/E5a.

In addition to the DFDC Collins prototype, a commercial Javad receiver available at DLR was used in the replay test. Initially, the correlator spacing and the bandwidth were selected to match the Collins prototype setup for a comparison of the results obtained. Next, this receiver was used to investigate the impact of the receiver parameters as it allows the modification of the correlator spacing and the bandwidth.

\section{RF recorder}

The baseband samples were recorded using the Syntony ECHO-R recorder, which records signals on three different phase synchronous channels. The channels can be freely chosen within a frequency range from $1164 \mathrm{MHz}-1610 \mathrm{MHz}$. For the project data on two different channels with center frequencies $1.175 \mathrm{GHz}$ for GPS L5/Galileo E5a and 1.575 for GPS L1/Galileo E1 and $50 \mathrm{MHz}$ bandwidth were recorded. The quantization was 16 bit.

\section{Replay device}

The replay of the RF-data was performed using the Averna RP6100 record \& replay system shown in Figure 4. The replay device provides two channels up to $40 \mathrm{MHz}$ bandwidth in complex baseband of which each is using 16 bits per I1 sample. The main features of the Averna RP-6100P are described in Table 1. 


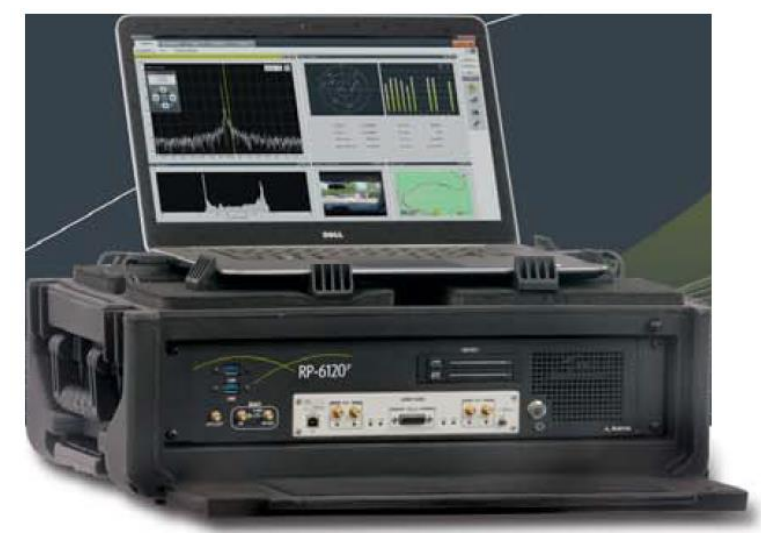

Figure 4 - Averna RP-6100 record and replay system

Table 1 - Averna RP-6100P Features

\begin{tabular}{|c|c|}
\hline \multicolumn{2}{|c|}{ Averna RP-6100P Features } \\
\hline Channels, Bandwidth & Two channels at 40 MHz each \\
\hline Recording capacity @ 1 Channel & 160 minutes at $40 \mathrm{MHz}$ \\
\hline Frequency range & $1-8 \mathrm{GHz}$ \\
\hline Bias-T (to power active GNSS Antenna) & One Bias-T 5.0 VDC at $100 \mathrm{~mA}$ \\
\hline Output resolution & $16 \mathrm{Bit}$ \\
\hline Dynamic range & $90 \mathrm{~dB}$ SFDR \\
\hline Drive view support & Camera + GPS position logging \\
\hline Recording storage & $8 \mathrm{~TB}$ \\
\hline
\end{tabular}

\section{MULTIPATH AND NOISE ERRORS FOR DIFFERENT PHASES OF FLIGHT}

Before discussing the obtained multipath models from this aircraft, an analysis of the multipath errors for different phases of flights is presented. For this analysis, the second flight (F0325) is chosen because it contains several landing and taxiing periods as previously detailed.

The multipath and noise error present on the code measurements were estimated using code-minus-carrier method which is described in detail in [3]. The choice of a cycle slip detector is an important step in the derivation of the final models. On one side, if not detected, the cycle slips affect the multipath estimation process (leading to a wrong carrier phase ambiguities estimate) and on the other side, the cycle slip detectors might exclude large jumps in the multipath. In order to be aligned with the processing of the airborne receivers, the detection of the cycle slips was based on the measurement-quality-monitor (described in [1] and [2]) and a limit on the carrier-to-noise values set to $29 \mathrm{~dB}-\mathrm{Hz}$ for L1/E1 signals and $27 \mathrm{~dB}-\mathrm{Hz}$ for L5/E5a signals.

Figure 5 shows the height above ground of this flight where it is visible when the aircraft was parked (black), taxiing and ground static (dark red) and when it was airborne (blue and green). The graph distinguishes also between heights of $300 \mathrm{~m}$ or less above ground (shown in blue) as this corresponds to the theoretical maximum height where ground reflections could affect the aircraft. For the analysis shown here it is only distinguished between air and ground data as the number of samples in the blue area (i.e. flying below $300 \mathrm{~m}$ ) is very small and thus no meaningful analysis of that period can be conducted separately. The taxiing data includes moving and stationary periods as they would occur during regular operations and are used to analyse the impact of the ground reflections on the multipath. 


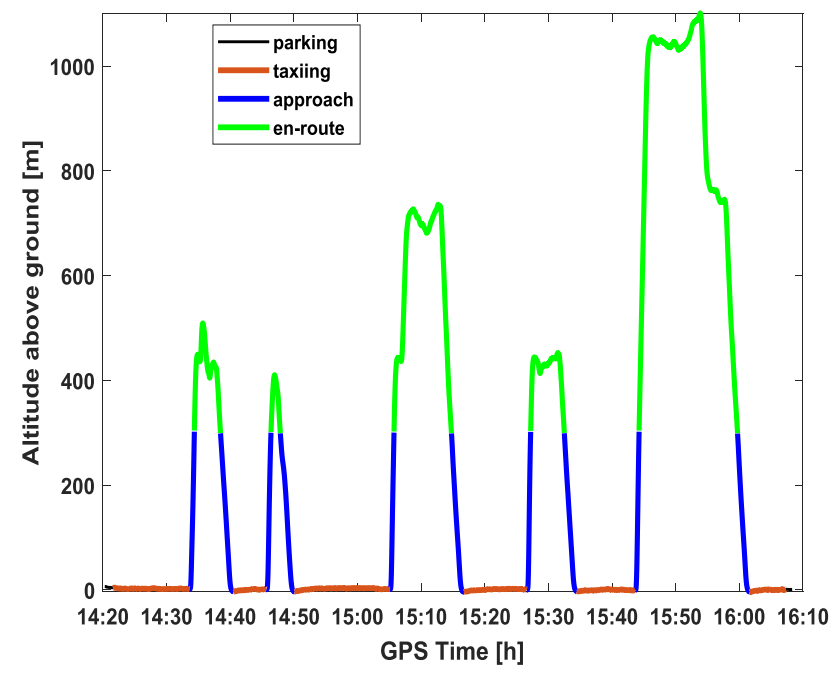

Figure 5 - Altitude above ground versus time for F0325

Figure 6 and Figure 7 show an evaluation of the unsmoothed multipath and noise error (top graphs) and 100 seconds smoothed multipath and noise errors over time for all available GPS and Galileo satellite for the L1/E1 and L5/E5a signals, respectively. In both figures, the yellow areas mark the periods when the aircraft was taxiing on ground (during moving and stationary periods) and the red areas mark the periods when the aircraft was at its parking position. During these periods marked with red no attitude data was available, but only GNSS measurements. Thus, these portions of data are not used in the derivation of the multipath models.

Looking at the unsmoothed multipath and noise errors on L1/E1 and L5/E5a it can clearly be observed that the L1 errors are significantly larger. However, after smoothing (the bottom plots in both figures) this difference shrinks and the errors are rather similar on both frequencies, being slightly smaller for L1/E1 signals. This behavior can be attributed to the specific antenna performance and is in line with the predicted multipath errors derived from the electromagnetic simulations which are shown in [8]. The larger errors on L1/E1 before smoothing can be explained by the increased receiver thermal noise errors which are very effectively smoothed out. The remaining errors after smoothing result from the antenna performance that is worse on L5/E5a compared with L1/E1.

Furthermore, in both figures the increase on the multipath error during taxiing compared to the periods when the aircraft was flying can be clearly observed. The increase is visible on both frequencies and both constellations. The increased residual errors in the taxiing periods also include static periods where the aircraft was holding near the runway. In these periods no movement occurred, and therefore no quick change in multipath that would be removed by smoothing was observed. Due to the limited number of samples during the taxi phase the results are only shown qualitatively in the plots above but no quantitative assessment of the difference between the phases was performed at this time. 


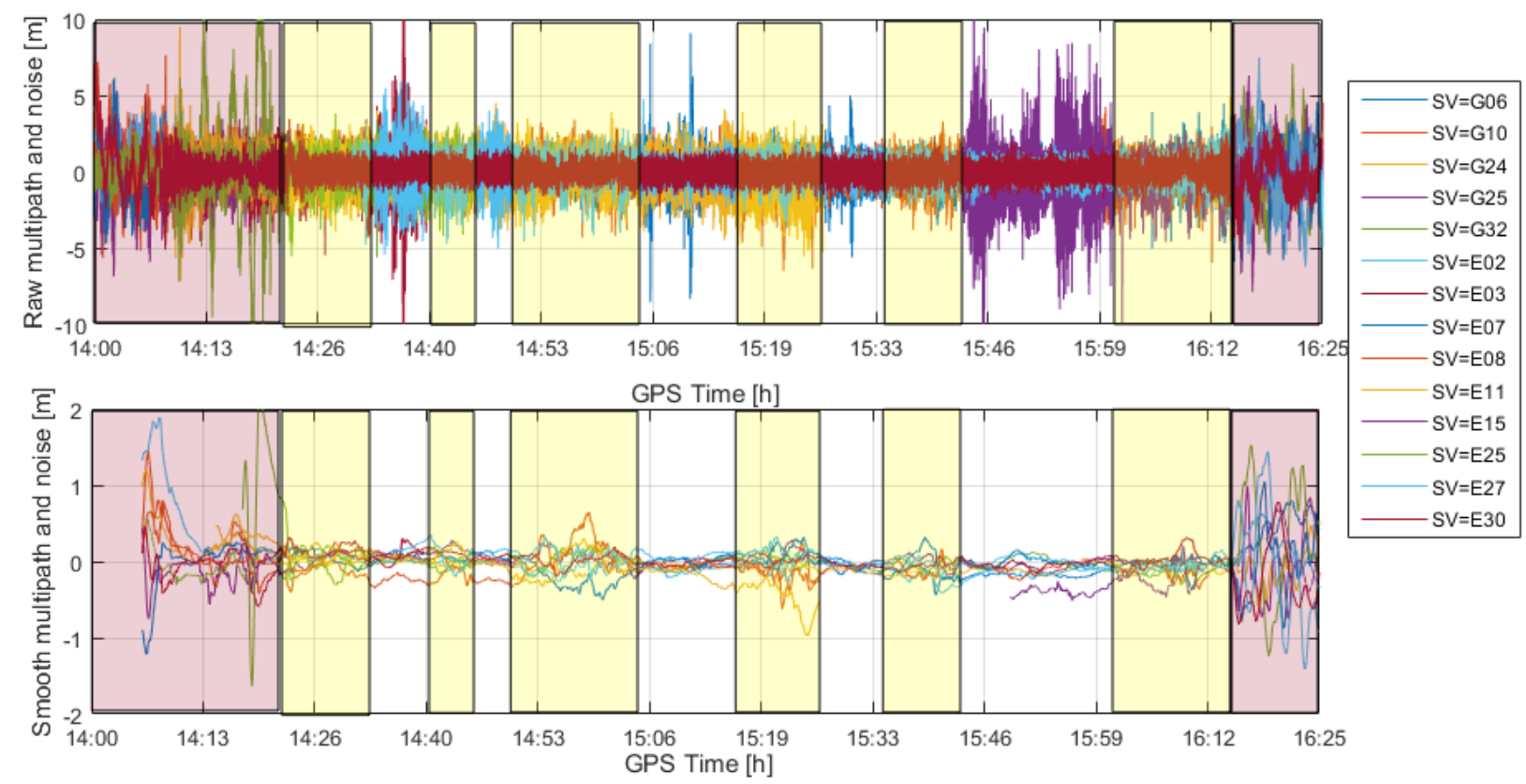

Figure 6 - Raw multipath and noise error versus time (top graph) and 100 seconds smoothed multipath and noise error (bottom) on L1/E1 for F0325 for GPS and Galileo satellites including also the data when the aircraft was parked. The yellow boxes mark the periods when the aircraft was on ground and the red boxes mark the parking periods

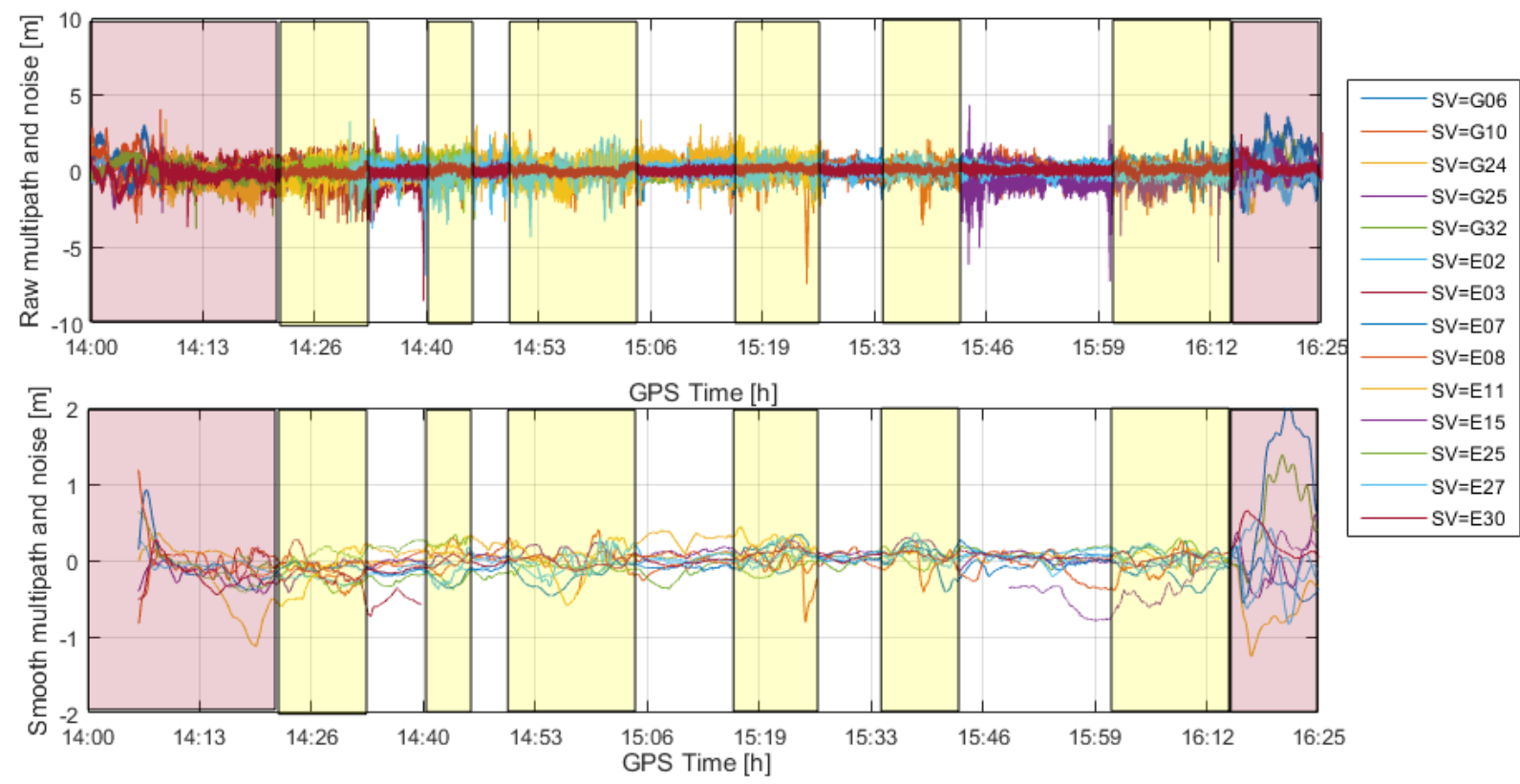

Figure 7 - Raw multipath and noise error versus time (top graph) and 100 seconds smoothed multipath and noise error (bottom graph) on L5/E5a for F0325 for GPS and Galileo satellites including also the data when the aircraft was parked. The yellow boxes mark the periods when the aircraft was on ground and the red boxes mark the parking periods 


\section{INITIAL RESULTS ON MULTIPATH}

The measurements replayed through the hardware receivers were used to derive the multipath models based on the following steps:

1. Estimation of the raw code multipath error using dual-frequency code-minus-carrier method.

2. Computation of elevation and azimuth angles in aircraft body frame coordinate system using the aircraft basic information and in a local level frame with respect to satellite elevation above the horizon.

3. Removal of the code errors due to the variation of the antenna group delay.

Previous work presented in [3] showed that the antenna-induced errors on the code measurements are different from the noise-like multipath and noise errors and proposed a methodology to separate these errors from multipath estimates. The antenna-induced errors for the entire hemisphere are shown in Figure 3. Based on the specific elevation and azimuth angles, the errors for each satellite are computed and subtracted from the estimated multipath and noise errors.

4. Smoothing of the estimated multipath errors using a smoothing time constant of 100 seconds. Within the project, only the errors in the filter steady state (after the smoothing filter converged) are characterized. We consider that the filter reaches the steady state after 3.6 times the smoothing time constant (e.g. 360 seconds for 100 seconds smoothing time) [9].

5. Selection of elevation bins and computation of the standard deviation of the multipath and noise errors for each elevation bin.

6. Overbounding of the data to safely bound the tail risk as described in [4]. This step is applied independently to each frequency and the ionospheric - free combination of the signals of the two frequencies.

7. No inflation due to the limited number of samples was performed. The number of samples collected was too small and the distribution of the samples across the elevations to uneven in order to obtain meaningful results.

Note that in this work the models describe a combination of the multipath and noise errors and a separation of the noise from the multipath errors will be addressed in future work. However, the multipath is correlated in time and will remain predominant after smoothing, while the noise is uncorrelated and will be smoothed out almost completely.

The RF-data collected during the flights conducted on the A321 aircraft were replayed through the dual-frequency dualconstellation Collins prototype receiver. The receiver was set up to use an early-minus-late correlator with a spacing of 0.1 chips for the L1/E1 signals and 1 chip for the L5/E5a signals. Attitude information from the basic instrumentation provided by Airbus was used to compute the elevation and azimuth angles in the aircraft body frame.

During the two flights on A321, around 17 satellite hours from GPS Block II satellites and 25 satellite hours from Galileo satellites were collected. Figure 8 shows a skyplot of the tracked GPS Block IIF satellites (left graph) and of the Galileo satellites (right graph), respectively.
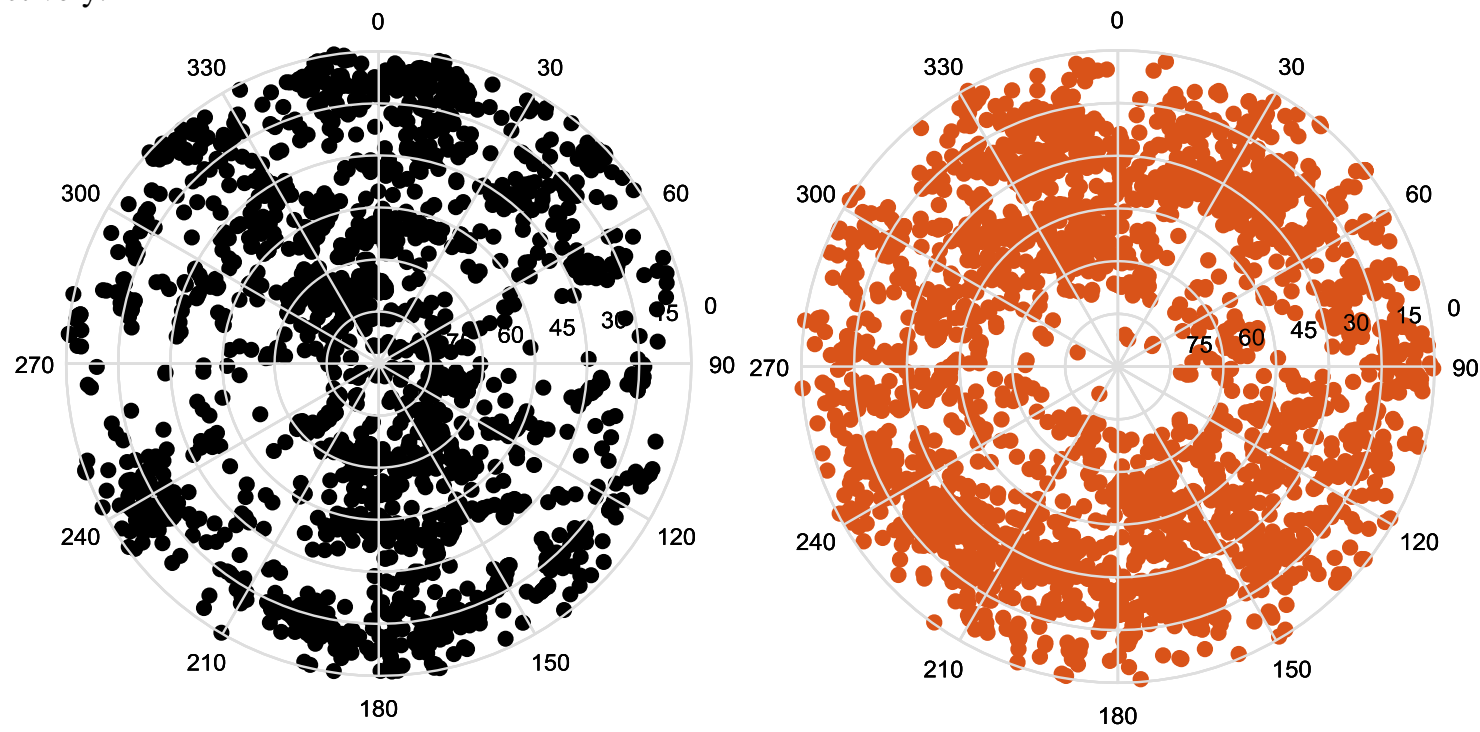

Figure 8 - Skyplot for the GPS tracked satellites (left plot) and Galileo tracked satellites (right plot) during the two flights conducted on Airbus A321 aircraft 
Figure 9 and Figure 10 show the comparison of the effect of the removal of the antenna errors induced by the group delay variations for GPS and Galileo respectively. The graphs show the standard deviations of the noise and multipath errors function of the satellite elevation in aircraft body frame for GPS L1/Galileo E1 with black lines and for GPS L5/Galileo E5a with green lines. The dashed lines represent the errors containing also the antenna error inside and the solid lines show the curves after the antenna errors removal.

It can be observed that the antenna removal had a more pronounced effect for low-medium elevations (below $50^{\circ}-60^{\circ}$ ) because the group delay variation is larger for these elevations. The effect is more pronounced on the L5/E5a signals because this antenna has worse performance and shows stronger variations on the L5 band compared with the L1. For the results presented next, this calibration has been performed and the curves refer to the multipath and noise errors without the contribution of the antenna.

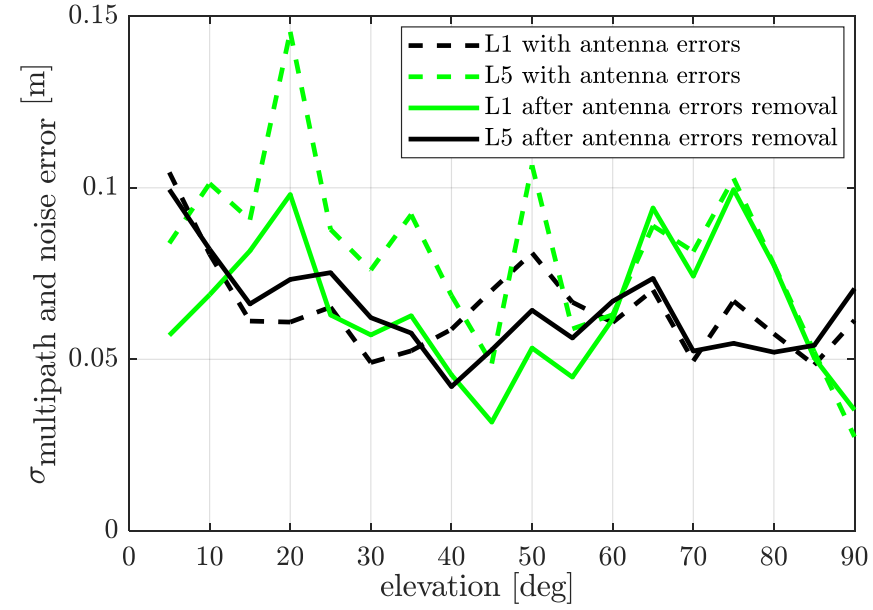

Figure 9 - Standard deviation of 100 seconds smoothed multipath and noise error for GPS L1 (black curves) and GPS L5 (green curves) containing the antenna-induced errors (dashed lines) and after removal of these errors (solid lines)

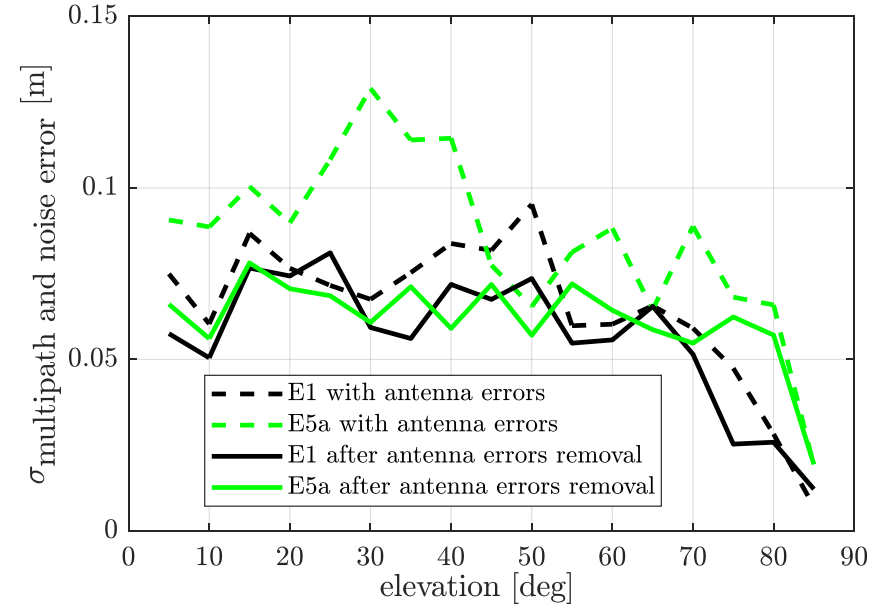

Figure 10 - Standard deviation of 100 seconds smoothed multipath and noise error for Galileo E1 (black curves) and Galileo E5a (green curves) containing the antenna-induced errors (dashed lines) and after removal of these errors (solid lines)

Figure 11 and Figure 12 show the overbounding multipath curves based on measurements from both A321 flights for GPS and Galileo. The dashed grey line in both figures represents the current GPS L1 airborne multipath model together with AAD-B noise curve. The curves refer to the errors after the antenna errors removal and are inflated to bound safely the risk in the tails.

Compared with the previous results derived from the DLR's A320 experimental installation (see [4]), the results show much lower levels of residual noise and multipath errors. This was to be expected as the experimental antenna on DLR's A320 is installed further to the back on the fuselage and is therefore subject to more multipath from the airframe. Furthermore, the aircraft is equipped with several other antennas and installations on the fuselage in the vicinity of the experimental GNSS antenna creating again more multipath than a standard installation would experience. Finally, the experimental antenna used is performing significantly worse in terms of multipath rejection capability and group delay variation than the antenna used for the Airbus A321 test flight.

It is interesting to note that the L5/E5a curves are slightly higher than the one derived for the L1/E1 signals. Previous studies have shown an improved performance and lower multipath and noise residual errors for the L5/E5a. However, as already shown and discussed in Figure 7, after smoothing, the difference between the two signals is similar with slightly better results for L1/E1 signals. This behavior can be attributed to the antenna that shows worse performance on the L5/E5a band compared with the L1/E1 band. Even if the antenna-induced errors are removed, the antenna multipath rejection capability has an important role in how much multipath is received ([8] and [10]). Furthermore, the L5/E5a BPSK(10) modulation has an improved multipath rejection capabilities for long range multipath (larger than 30 meters). However, on the aircraft short range multipath is expected with the further away reflector being the tail which is less than 30 meters away from the antenna. For short range multipath, the BPSK(10) has similar expected code errors with those of the L1 BPSK(1) and E1 BOC $(1,1)$ modulations. 
Similar to the results derived from the experimental DLR installation, the curves do not show a strong elevation dependency. Furthermore, no large difference has been observed when considering the satellite angle in the local frame or the level frame (above horizon).

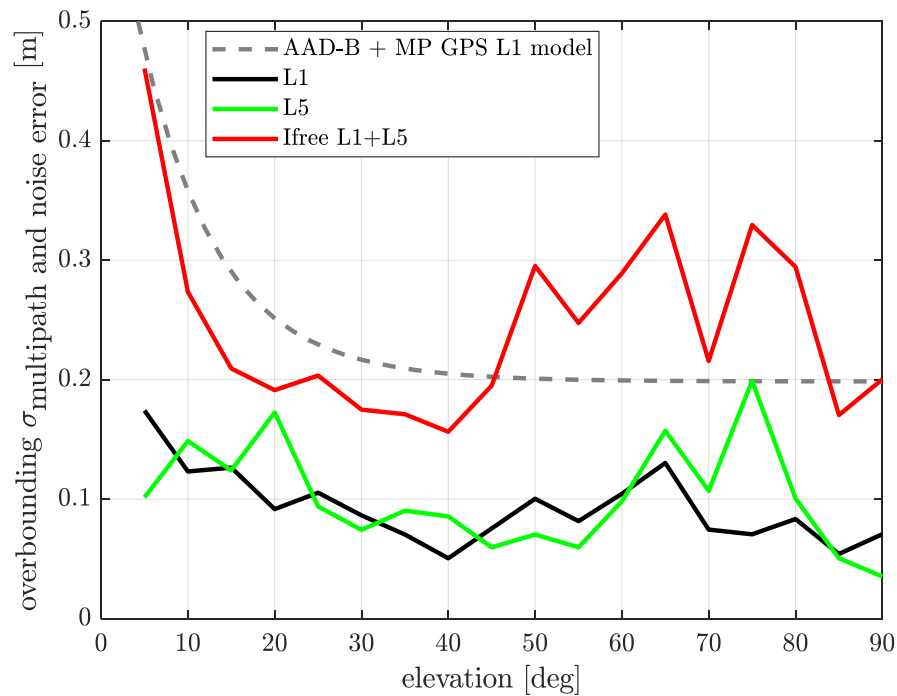

Figure 11 - Overbounding standard deviation of the 100 seconds noise and multipath error for GPS L1, GPS L5 and Ifree combination based on approx. 5 hours of flight data recorded and replayed through DFDC Collins MMR after 100 seconds smoothing

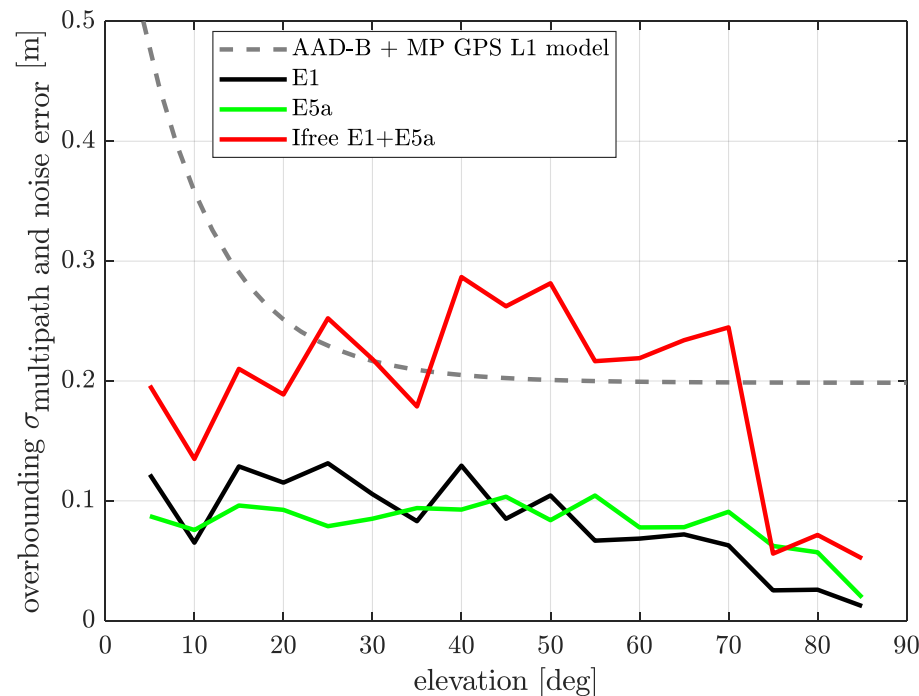

Figure 12 - Overbounding standard deviation of the 100 seconds noise and multipath error for Galileo E1, Galileo E5a and Ifree combination based on approx. 5 hours of flight data recorded and replayed through DFDC Collins MMR after 100 seconds smoothing

\section{IMPACT OF RECEIVER PARAMETERS}

Another aspect that was investigated within the project is the impact of the receiver design space in terms of correlator spacing and bandwidth. This study was carried out using a commercial Javad receiver. The reason for using Javad receivers is that it allows the modification of the front-end bandwidth in addition to the modification of the correlator spacing.

In order to validate that the obtained results from the Javad receiver are representative, the RF-data from one flight was replayed in parallel through the Javad receiver and the DFDC Collins prototype receiver. For this test, both receivers were set up with similar configuration in terms of correlator spacing ( 0.1 chips for L1/E1 and 1 chip for L5/E5a). Figure 13 and Figure 14 show a comparison of the obtained standard deviation of the 100 seconds smoothed multipath and noise errors for GPS and Galileo L1/E1(black curves) and L5/E5a (green curved) from the two receivers. The multipath curves are plotted as a function of elevation in aircraft body frame. The solid lines represent the results obtained using measurements from Collins DFMC prototype receiverand the dashed line the results derived from the Javad receiver.

For both constellations, the curves from the two receivers match well and the differences are within few centimeters. For GPS, the difference on the multipath curves derived for the L5 signal is slightly larger but stays within 2-3 cm. For the Galileo signals, the difference between the two receivers is very small, below $1 \mathrm{~cm}$ for almost all elevation bins, except the $70^{\circ}-75^{\circ}$ where it reached a maximum of $4 \mathrm{~cm}$. The measurements from the two receivers were synchronized, however small differences in the available data are present which may explain the different values.

Based on these results, we can conclude that the behaviour of the Javad receiver is very similar to the one of the Collins DFMC prototype receiver and thus study of the impact of the correlator spacing and bandwidth using the Javad receiver can be considered representative for what is to be expected from an avionics receiver. Further validation of this assumption will be done in future work. 


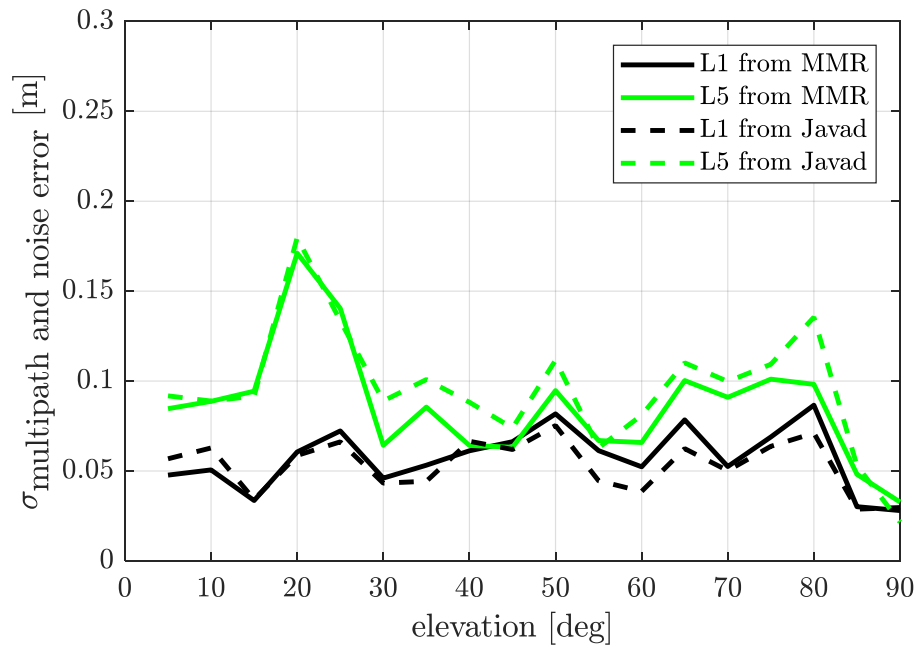

Figure 13 - Standard deviations of the 100 seconds smoothed multipath and noise error function of elevation for GPS L1 (black line) and GPS L5 (green lines). The solid lines represent the results obtained

from the DFDC Collins prototype receiver and the dashed lines represent the results from the Javad receiver

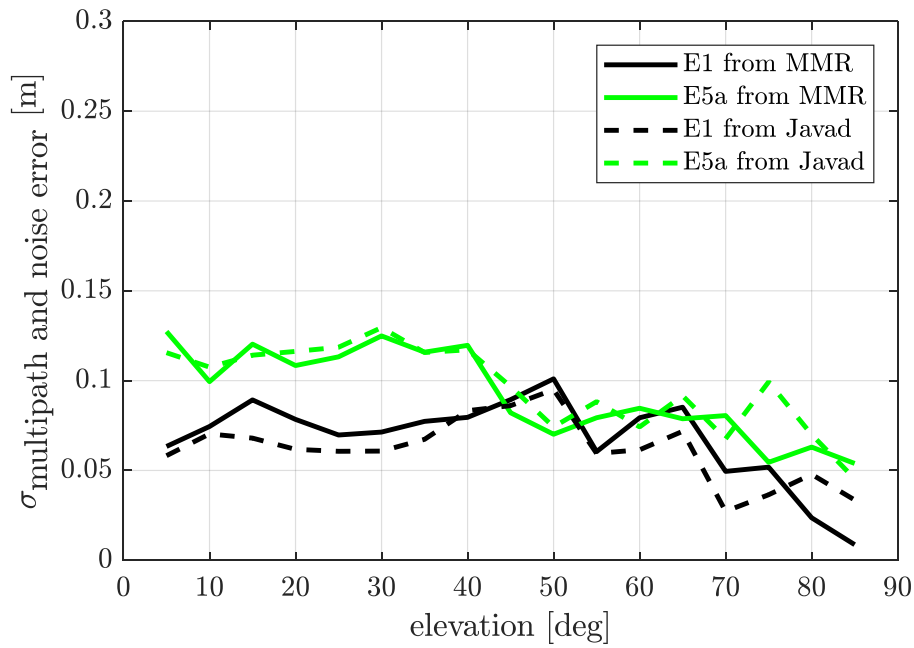

Figure 14 - Standard deviations of the 100 seconds smoothed multipath and noise error function of elevation for Galileo E1 (black lines) and

Galileo E5a (green lines). The solid lines represent the results obtained from the DFDC Collins prototype receiver and the dashed lines represent the results from the Javad receiver

The multipath and noise error is expected to change when different configurations are used. The current dual-frequency dualconstellation airborne specifications define the allowed regions for combinations of receiver bandwidth and early-minus-late correlator spacing for Galileo E1 and L5/E5a signals. For these studies, the values given in [11] and shown in Table 2 are used.

Table 2 - Receiver design space for DFMC SBAS taken from [11]

\begin{tabular}{|c|c|c|c|c|}
\hline & \multicolumn{2}{|c|}{ Galileo E1C signal (CBOC(6.1)) } & \multicolumn{2}{|c|}{$\begin{array}{l}\text { Galileo E5a and GPS L5 signal } \\
(B P S K(10))\end{array}$} \\
\hline & reference & user & reference & user \\
\hline $\begin{array}{l}\text { Tracking } \\
\text { technique }\end{array}$ & $\begin{array}{l}\text { EML (BOC(1.1) } \\
\text { local replica) }\end{array}$ & $\begin{array}{l}\text { EML }(B O C(1.1) \\
\text { local replica) }\end{array}$ & $\begin{array}{l}\text { EML (BPSK }(10) \\
\text { local replica) }\end{array}$ & $\begin{array}{l}\text { EML (BPSK }(10) \\
\text { local replica) }\end{array}$ \\
\hline $\begin{array}{l}\text { Correlator } \\
\text { spacing }\end{array}$ & 0.1 chip & $\begin{array}{l}0.08 \text { and } \\
0.12 \text { chip }\end{array}$ & 1 chip & $0.8,1.2$ chip \\
\hline $\begin{array}{l}\text { Pre-correlation } \\
\text { bandwidth } \\
\text { (double-sided) }\end{array}$ & $24 \mathrm{MHz}$ & $\begin{array}{c}12,14,16,18,20, \\
22,24 \mathrm{MHz}\end{array}$ & $24 \mathrm{MHz}$ & $\begin{array}{c}12,14,16,18,20, \\
22,24 \mathrm{MHz}\end{array}$ \\
\hline $\begin{array}{l}\text { Equivalent } \\
\text { reception filter }\end{array}$ & \multicolumn{4}{|c|}{$\begin{array}{l}4 \text { filters are tested ( } 6^{\text {th }} \text {-order Butterworth, } 0 \text {-group delay resonator, } 150 \mathrm{~ns} \\
\text { differential group delay resonator, } 150 \mathrm{~ns} \text { differential group delay } 6^{\text {th }} \text {-order } \\
\text { Butterworth) to estimate differential tracking error. } \\
\text { Only the } 6^{\text {th }} \text {-order Butterworth filter is applied to the reference to estimate the } \\
\text { absolute tracking error. }\end{array}$} \\
\hline
\end{tabular}

Based on these specifications, the four corners of the bandwidth-correlator spacing design space were chosen :

- Configuration 1: $12 \mathrm{MHz}$ bandwidth - 0.08 chips correlator spacing for L1/ 0.8 chips correlator spacing for L5

- Configuration 2: $12 \mathrm{MHz}$ bandwidth - 0.12 chips correlator spacing for L1/ 1.2 chips correlator spacing for L5

- Configuration 3: $23 \mathrm{MHz}$ bandwidth - 0.08 chips correlator spacing for L1/ 0.8 chips correlator spacing for L5

- Configuration 4: $23 \mathrm{MHz}$ bandwidth - 0.12 chips correlator spacing for L1/ 1.2 chips correlator spacing for L5 
The RF-data from the first flight conducted on A321 was replayed four times to the receiver configured in all four combinations. The advantage of replayed the RF-data through the same receiver type is that all the other effects are the same (e.g. satellite geometry, antenna) and it allows to isolate the effect of the receiver configuration on the derived multipath curves.

Figure 15 shows the comparison of the standard deviations of the unsmoothed multipath and noise error as a function of the satellite elevation angle in the aircraft body frame for the four different configurations for L1 (top graph), L5 (middle graph) and the Ifree combination (bottom graph).

For GPS L1, there is a noticeable difference between the different cases. The lowest curve (blue curve with 'diamond' marker) is obtained from the measurements collected with the narrowest bandwidth $(12 \mathrm{MHz})$ - narrowest correlator spacing $(0.08$ chips for L1) and the largest error results from the $23 \mathrm{MHz}$ bandwidth with 0.12 correlator (purple line with ' $v$ '). This can be explained by the fact that the unsmoothed errors are dominated by the impact of the receiver noise, which is directly proportional to the receiver bandwidth and correlator spacing. For the L5 signal, the difference between the different cases is very small and only visible for low elevations. This is because the L5 signal with the BPSK(10) modulation has a much lower noise error (due to the higher chipping rate) compared with the L1 signal.

The dominance of the noise error in the unsmoothed curves is also confirmed by the results obtained after applying the smoothing filter. The standard deviations of the noise and multipath error obtained after 100 second smoothing for GPS signals are shown in Figure 16 in a similar manner with the previous figure. It can be observed that after smoothing the obtained curves for the different cases are very similar and the difference reduces significantly, being almost negligible.

Figure 16 and Figure 17 show the results obtained for Galileo signals, for the unsmoothed multipath and noise error and after 100 seconds smoothing, respectively. A very similar behavior with GPS can be observed: the difference between the cases is pronounced for the unsmoothed errors and decreases significantly after smoothing. The difference on the unsmoothed multipath and noise error is larger on the E1 signals reaching up to $40-50 \mathrm{~cm}$ while on the E5a signal it is in the order of few centimeters. The large difference on E1 yields a difference of up to $70-80 \mathrm{~cm}$ between the unsmoothed noise and multipath of the Ifree combination for the different cases. In the smoothed errors, the differences for E1, E5a and Ifree stay very small.

As the four combinations of correlator spacing-receiver bandwidth were selected as the corners of the receiver design space, it is expected that the results from any other combination would lie within the limits defined by these cases.

From this study the following conclusions can be drawn and will be validated in further studies within the project:

- after 100 seconds smoothing the multipath and noise curves for the different values of the correlator spacing and bandwidth (within the DFMC design space) look very similar and thus one single model should be sufficient to cover the entire design space

- the change in the receiver parameters has a visible impact on the unsmoothed multipath and noise error due to the dominance of the high frequency noise and the final choice of a model for the unsmoothed errors will need to take into account these differences. 
GPS L1
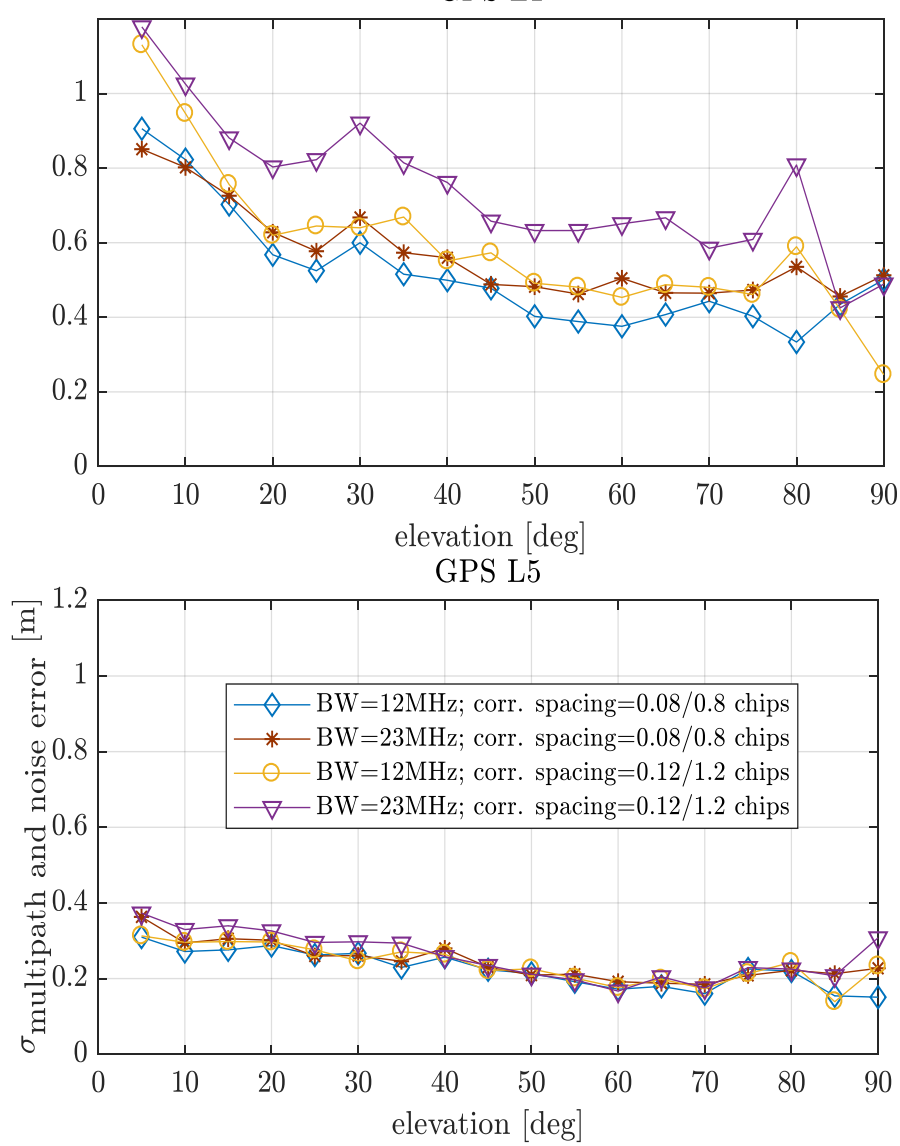

GPS Ifree $\mathrm{L} 1+\mathrm{L} 5$

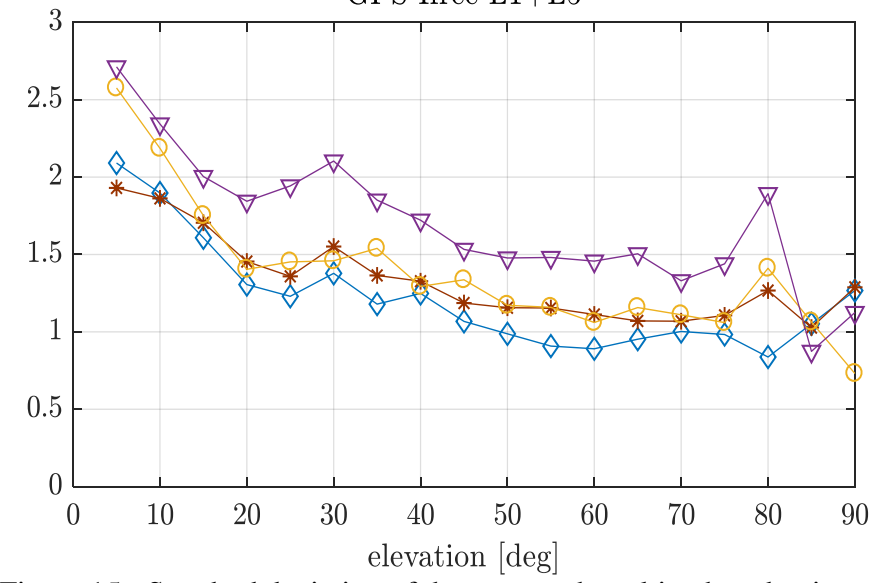

Figure 15 - Standard deviation of the unsmooth multipath and noise errors function of the elevation in the aicraft body frame for different receiver bandwidth-correlator spacing combination for GPS L1 (top graph), GPS L5 (middle plot) and GPS Ifree combination (bottom plot)
GPS L1

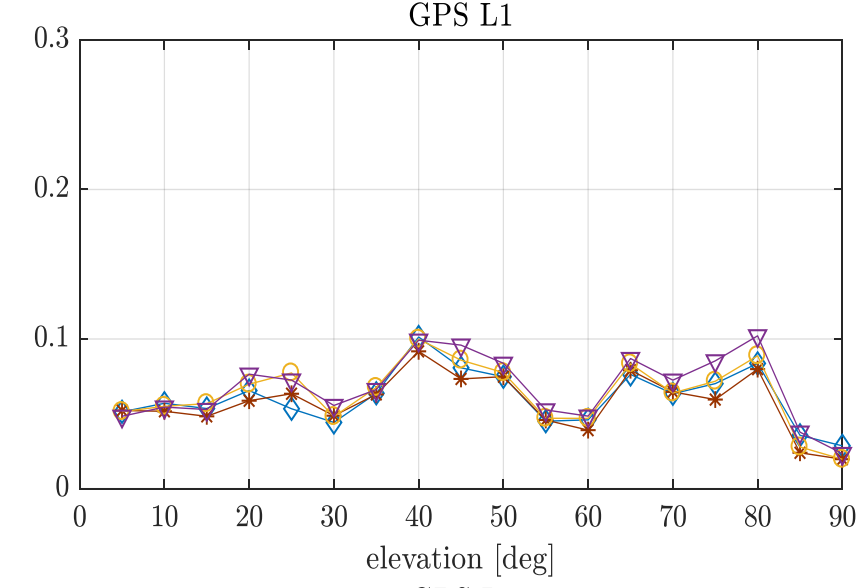

GPS L5

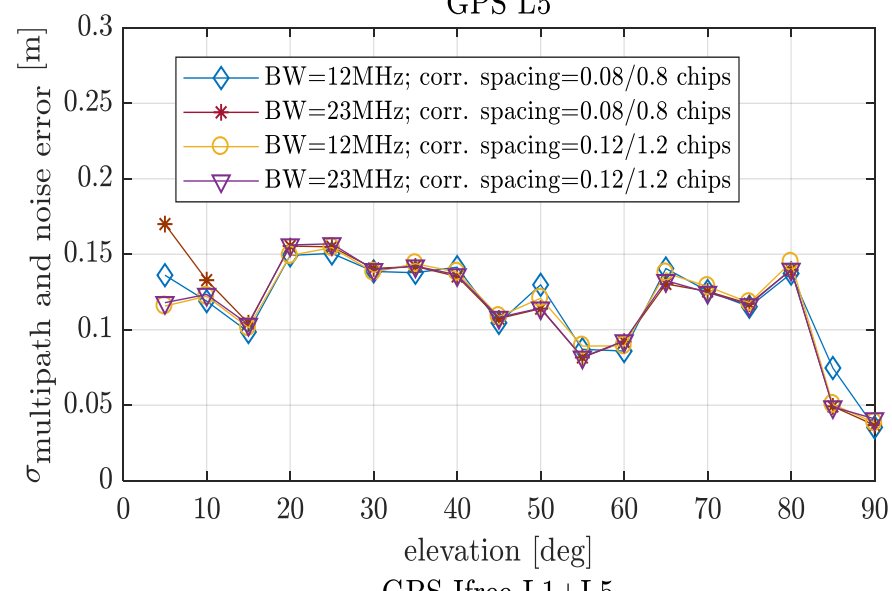

GPS Ifree $\mathrm{L} 1+\mathrm{L} 5$

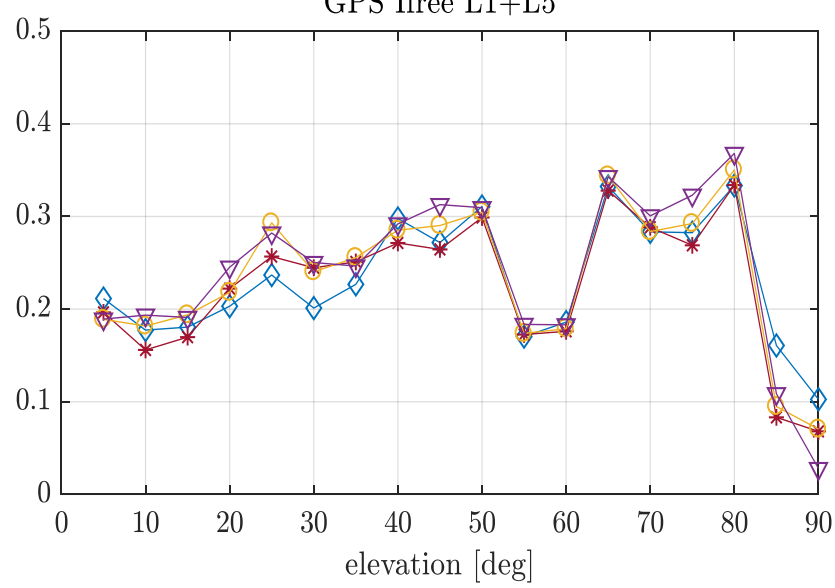

Figure 16 - Standard deviation of the 100 seconds smoothed multipath and noise errors function of the elevation in the aircraft body frame for different receiver bandwidth-correlator spacing combination for GPS L1 (top graph), GPS L5 (middle plot) and GPS Ifree combination 

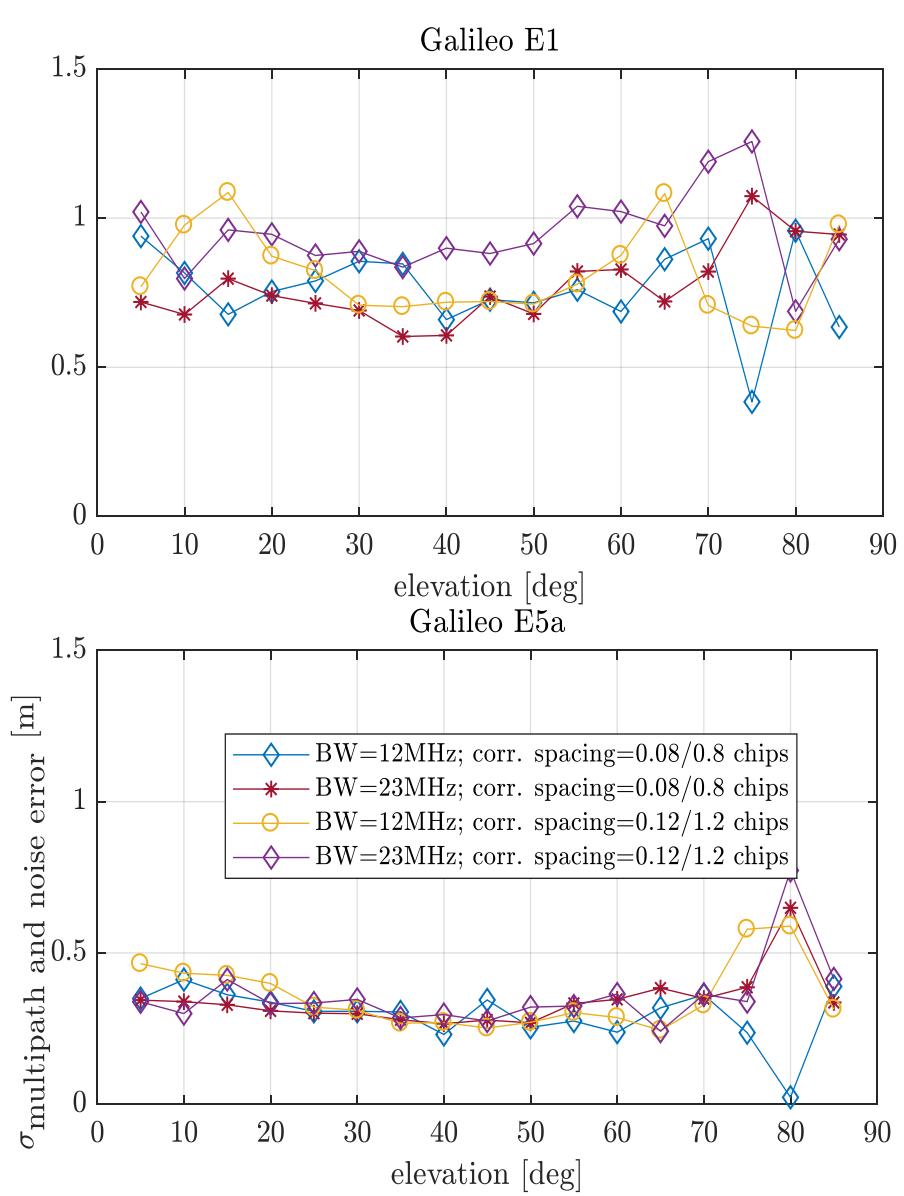

Galileo Ifree E1+E5a

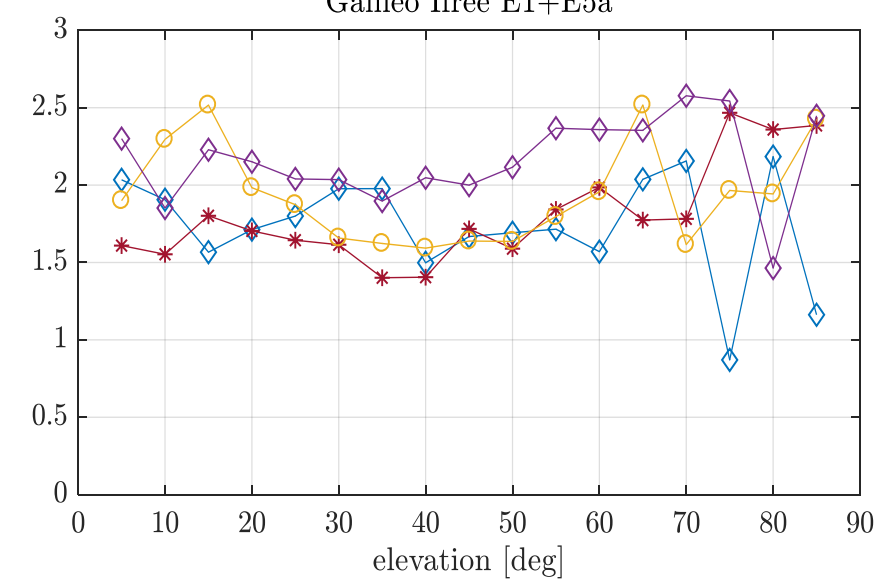

Figure 17 - Standard deviations of the unsmooth multipath and noise errors function of the elevation in $\mathrm{A} / \mathrm{C}$ body frame for different receiver bandwidth-correlator spacing combinations for Galileo E1 (top graph), Galileo E5a (middle plot) and Galileo Ifree combination (bottom plot)
Galileo E1
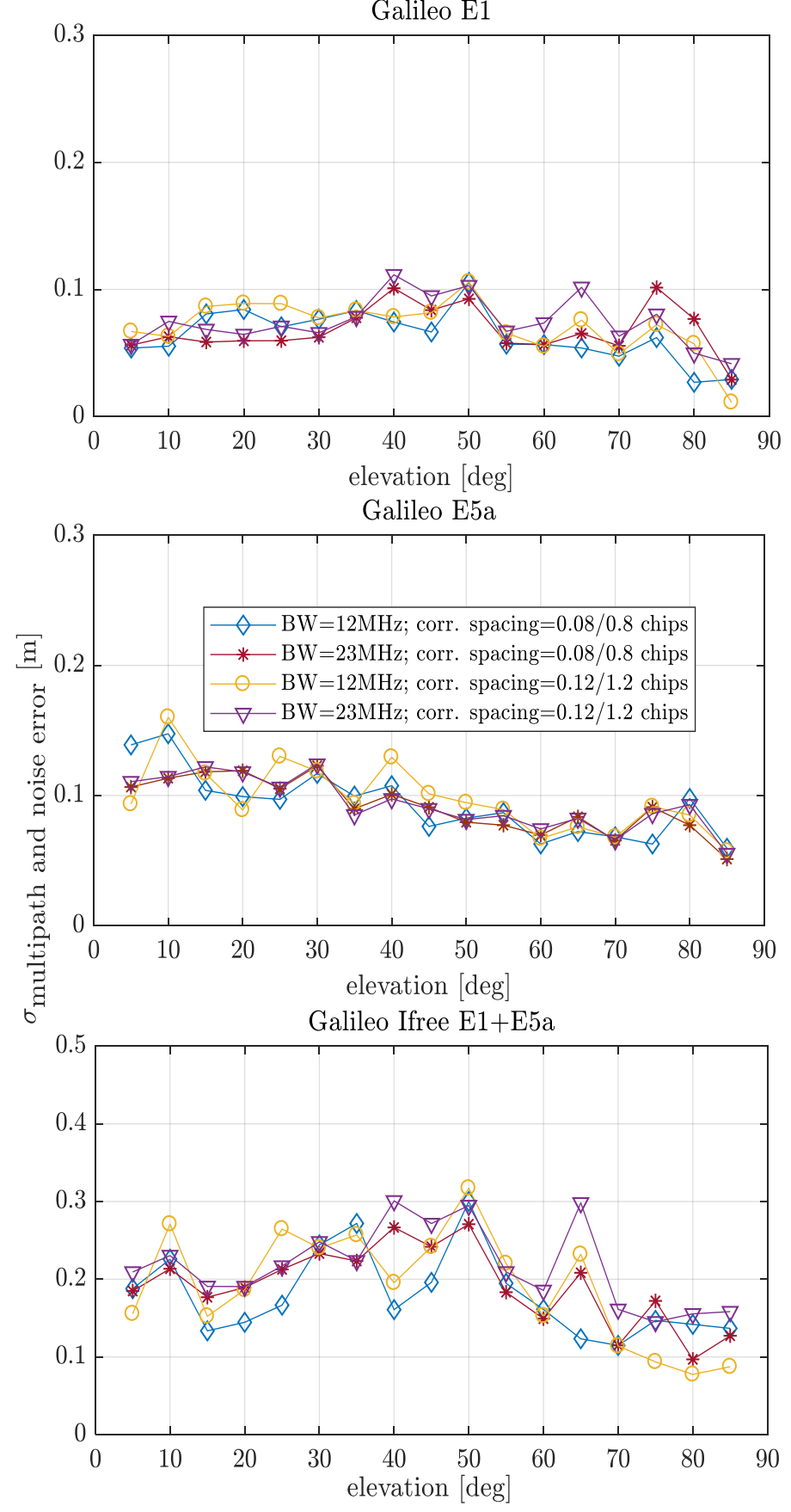

Figure 18 - Standard deviations of the 100 second smooth multipath and noise errors function of the elevation in $\mathrm{a} / \mathrm{c}$ body frame for different receiver bandwidth-correlator spacing combinations for Galileo E1 (top graph), Galileo E5a (middle plot) and Galileo Ifree combination (bottom plot) 


\section{CONCLUSION}

In this paper we presented initial results of the DUFMAN project resulting from a detailed analysis of the first batch of flight data collected with an Airbus A321. Approximately 5 hours of GNSS data were recorded with a MOPS compliant GNSS antenna installed in its designated location. The data was replayed to a prototype DFMC avionics receiver and in comparison to a commercial receiver that was previously used in DLR's flight test campaigns.

The most important results in terms of developing a DFMC multipath model were:

- $\quad$ Noise and multipath on the L1 and E1 signals before smoothing are significantly larger than on L5 and E5a

- This difference vanishes after smoothing. In our case the performance of L5/E5a after smoothing is slightly worse than on L1/E1.

- Group delay variation of the antenna may introduce significant code tracking errors that have to be either calibrated, or accounted for in integrity considerations or specified to be small enough to have no significant impact on the airborne performance models

- The level of noise and multipath after $100 \mathrm{~s}$ smoothing is significantly smaller than in previous flight trials by DLR due to the representative installation

- The choice of correlator spacing and receiver bandwidth according to the proposed SBAS MOPS has an impact on the raw GNSS measurements and causes significant differences between the signals. This difference, however, is removed almost completely by 100 seconds smoothing.

Within the DUFMAN project more data with different aircraft will be collected to obtain results from a variety of representative aircraft, as well as a somewhat statistically representative number of samples for deriving noise and multipath models.

\section{REFERENCES}

[1] RTCA DO-253D, " Minimum Operational Performance Standards for GPS Local Area Augmentation System Airborne Equipment", 2017

[2] WAAS Minimum Operational Performance Specification (MOPS), RTCA document DO-229D

[3] M.-S. Circiu, S. Caizzone, M. Felux, C. Enneking, M. Meurer, "Improved Airborne Multipath Modelling", Proceedings of ION GNSS+ 2018 , Miami, FL, USA, September 2018

[4] M. Felux, M.-S. Circiu, S. Caizzone, C. Enneking, F. Fohlmeister, M. Rippl, "Towards Airborne Multipath Models for Dual Constellation and Dual-frequency GNSS”, Proceedings of the 2019 International Technical Meeting of The Institute of Navigation, Reston, VI, UDA, January 2019

[5] DO-373 - Minimum Operational Performance Standards for GNSS Airborne Active Antenna Equipment for the L1/E1 and L5/E5a Frequency Bands, RTCA 2018

[6] Caizzone S., Circiu, M.S., Elmarissi W., Enneking C., Felux M., Yinusa K. „Effect of Antenna Pattern Uniformity on the Pseudorange Tracking Error" Proceedings of the ION GNSS+ 2017, Portland, Oregon, USA

[7] Caizzone S., Circiu M-S., Elmarissi W., Enneking C., Felux M., Yinusa K. "Antenna influence on Global Navigation Satellite System pseudorange performance for future aeronautics multifrequency standardization", Journal NAVIGATION 2019; Volume 66, pages 99-116, doi:https://doi.org/10.1002/navi.281

[8] Caizzone S., Circiu M.-S., Elmarissi W., Enneking C., Winterstein A., „Airborne Antenna and Multipath Error Characterization for DFMC Error Standardization “, Proceedings of ION GNSS+ 2019, September 2019, Miami, US

[9] Eurocae ED-114A, Minimum Operational Performance Specification for Global Navigation Satellite Ground Based Augmentation System Ground Equipment to Support Category I Operations, Eurocae Std. ED-114A, Mar. 2013

[10] Caizzone S., Circiu M.-S., Elmarissi W., Enneking C, Felux M., Yinusa K.: "Multipath Rejection Capability Analysis of GNSS Antenna", ION GNSS+ 2018, Miami, FL, USA

[11] Mabilleau M., “Galileo Evil Wave Form Model Methodology”, NSP JWGs/3-IP/35 